\title{
Within-category discriminations in speech perception
}

\author{
VICKI L. HANSON \\ University of Oregon, Eugene, Oregon 97403
}

\begin{abstract}
The assumption that listeners are unaware of the highly encoded acoustic properties which lead to phoneme identification is questioned in the present study. It was found that some subjects can make use of small differences in voice onset time when making within-category discriminations. Subjects who can use these auditory features do so both implicitly (in a phonetic match task) and deliberately (in a physical match task). Results also indicate that some type of parallel process model is needed to account for the processing of auditory and phonetic information.
\end{abstract}

One characteristic of categorical speech perception is that the auditory information needed for making within-category discriminations is not available to listeners (Liberman, Cooper, Shankweiler, \& Studdert-Kennedy, 1967; Liberman, Harris, Hoffman, \& Griffith, 1957). For example, voice onset time (VOT) and formant transitions are acoustic dimensions used by listeners when processing speech. According to the categorical hypothesis, listeners are unable to use these auditory features to discriminate among different representations of the same phoneme. Support for this theory came from discrimination studies employing the ABX paradigm in which three speech sounds were successively presented and listeners judged whether the third sound was identical to the first or the second. This paradigm has been questioned, however, because it is heavily dependent upon memory (Pisoni, 1971, 1973). Studies placing less load on memory have found evidence that acoustic information can influence judgments within a category (Barclay, 1972; Pisoni \& Lazarus, 1974; Pisoni \& Tash, 1974).

Pisoni and Tash (1974) required listeners to respond "same" in a matching paradigm if both syllables of a stimulus pair belonged to the same phonetic category and to respond "different" if the two syllables belonged to different phonetic categories. Reaction times showed that the listeners were faster at responding "same" to two identical stimuli than they were at responding "same" to two instances of the same phonetic category that were acoustically different. Pisoni and Tash (1974) also found that the greater the dissimilarity in physical stimuli in different categories, the faster the RT.

This paper is based upon a master's thesis submitted to the Graduate School of the University of Oregon. I am very grateful to Michael Posner and Steven Keele for their helpful advice throughout this project and their comments on early drafts of the manuscript.
For example, RTs to different phonemic stimuli differing in 20-msec VOT for the bilabial stops were longer than RTs to phonemes with a $60-\mathrm{msec}$ VOT difference.

Wood (1974) reported evidence indicating that auditory and phonetic information is extracted in parallel. He varied auditory (pitch) and phonetic (place of articulation) features of speech either one at a time, orthogonally, or in a correlated manner so that the two features presented redundant information. The listener's task was to identify the value of a target dimension on each trial. In the control condition, RTs to identify the auditory features were not statistically different from the time to identify phonetic features. The fastest RTs for identification were in the correlated condition. If perception of the syllables were strictly a serial process, then RTs for the correlated conditions in Wood's study would have been no faster than RTs for the control conditions. Serial processing predicts that auditory processing always precedes phonetic processing, so there could be no redundancy gain.

Pisoni and Tash (1974) proposed a three-stage model to account for the processing of auditory and phonetic information in the phoneme matching task they used. As compatible with Wood (1974), they suggested that in the initial stage of processing, information about both auditory and phonetic properties of the syllables are encoded. In Stage 2 of processing, decisions about the physical similarity occur. The listener evaluates the acoustic difference against his criteria for a "same" and "different" stimulus; stimuli meeting such criteria are discriminated at this stage. For values of intermediate acoustic difference, a third stage of processing is necessary; at this level, phonetic judgments are made. Thus, in this model, the comparisons of auditory and phonetic information are processed serially.

The present study examined listeners' processing and use of an acoustic dimension. Each subject was 
given three separate tasks, and RT as well as accuracy was measured in all cases. The three tasks were a phoneme identification task for determining each subject's phonetic boundary, a phonetic match task similar to that used by Pisoni and Tash (1974), and a physical match task. There were three main issues.

First, the present study serves as a replication of Pisoni and Tash (1974), who demonstrated that acoustic information affects phonetic decision time. The physical match task will take the issue one step further. It is conceivable that the magnitude of acoustic differences would affect phonetic judgments even though listeners could not directly perceive the difference. That is, acoustic factors may affect the rate at which phoneme information becomes available to the listeners even though the listener is unable to deliberately use this auditory information. Thus, the physical match task is used in this study to determine whether listeners can directly perceive auditory features which lead to phoneme identification. Barclay (1972) has reported evidence suggesting that listeners are able to deliberately use this auditory information.

Second, what is the relation between implicit and deliberate use of auditory information? If individual subjects show ability to use this information implicitly, will these same subjects also be able to use it deliberately?

The final question deals with the issue of serial vs. parallel processing of auditory and phonetic information. Does crossing the phonetic boundary influence RTs when making physical matches? Different predictions are made by the two models. A serial model would predict that auditory information would be processed before phonetic information and therefore the phonetic boundary should not influence responding. A parallel model would predict that the redundant information provided by crossing the boundary would speed physical match decisions.

\section{METHOD}

\section{Experiment 1}

Stimuli and apparatus. The stimuli were 13 synthetic $\mathrm{CV}$ syllables. The syllables consisted of a bilabial stop, $/ \mathbf{b} /$ or $/ p /$, followed by the steady state vowel $/ a /$. The stop consonants spanned the VOT continuum from -5 - to +55-msec VOT in steps of $5 \mathrm{msec}$. Each syllable had a 50 -msec transitional period followed by a steady state portion of $250 \mathrm{msec}$. Stimuli were prepared at Haskins Laboratories and were then digitized on a PDP-9 computer for preparation of experimental tapes.

Subjects were seated in a soundproof booth, and stimuli were presented via headphones. Experimental tapes were played at a comfortable listening intensity on a Revox Model A77 tape recorder located in an adjacent room.

Experimental materials. The identification task consisted of 10 presentations of each stimulus. Trials were presented at the rate of one CV syllable every $2300 \mathrm{msec}$, with RT measured from the onset of the syllable.
The duration of each trial in both the phonetic and physical match tasks was $2,850 \mathrm{msec}$. On each trial, there were two syllables separated by an ISI of $570 \mathrm{msec}$, and RT was measured from the onset of the second stimulus.

In the phonetic match task, an attempt was made to equate the number of trials on which pairs of stimuli from the same and different phonetic categories occurred. The phonetic boundary for the VOT continuum generally has been found to be around 25- or 30-msec VOT for English speakers (Lisker \& Abramson, 1970; Pisoni \& Tash, 1974). For purposes of audio tape preparation, all stimuli at 25 -msec VOT or less were considered to be the voiced stop $/ b /$. All stimuli with VOT greater than this were considered to be the voiceless stop $/ p /$. Because individuals differ, the phonetic boundaries for the subjects in this experiment may not be the same as the average boundaries established in prior studies. Therefore, the number of "same" and "different" responses in this phonetic match task may not be equal for all subjects.

For different phonetic category trials in the phonetic match task, each test block contained one comparison at three, five, and seven steps for each stimulus value where these comparisons were possible. In addition, each block contained two one-step comparisons. For same phonetic category trials, each stimulus was paired once with itself and once with all possible five-step comparisons in each test block. Four one-step and six three-step same-category comparisons were also included in each test block.

For every block in the physical match task, each stimulus value was paired three times with itself and once each with stimuli one, three, and five steps removed on the acoustic continuum. Using the same phonetic boundary defined for the phonetic match task, for nonidentity trials in the physical match task, there were 26 trials from the same phonetic category and 13 test pairs from different phonetic categories.

Two experimental tapes were prepared as follows:

The first tape contained 130 practice identification $/ \mathrm{ba} /-/ \mathrm{pa} /$ trials followed by 130 test trials. Following the identification task, 2 blocks of practice trials for the phonetic match task were contained on the tape. Each block contained 64 trials. The last part of the tape contained 10 test blocks of 64 trials each for the phonetic match task.

The second tape contained only blocks of trials for the physical match task. The tape contained 2 practice blocks followed by 10 test blocks. Each block consisted of 78 trials.

Design. Each subject listened to both experimental tapes. The only between-subject factor in this experiment was order of task presentation. Half the subjects were first tested on the tape which contained the identification and phonetic match task, while the other half of the subjects first were tested on the physical match tape. For both these experimental groups, the identification task always immediately preceded the phonetic match task.

Procedure. The subjects were instructed that they would be hearing synthesized speech. In the identification task, they were instructed that they would be hearing either /ba/ or /pa/ and were asked to press one of two response keys to indicate which of these two syllables they heard on each trial.

In the phonetic match task, the subjects were instructed to respond "same" if both members of the trial pair contained the same consonant. If the consonants differed in the pair, then the subjects were to respond "different."

In the physical match task, the subjects' instructions were to respond "same" only if both consonants in the pair were identical. The subjects were to respond "different" if there was any acoustic difference in the two consonants, even if the same consonant was represented in both syllables of the stimulus pair. Notice that subjects who were tested in the physical match task first were not told which phonemes were represented by the synthetic speech syllables.

Subjects responded by pressing one of two response keys with their index fingers. In the identification task, one key was used for 
the /ba/ response and the other key was used for the /pa/ response. The response keys were counterbalanced across subjects for hand of response. Similarly, in the matching tasks, "same" and "different" response keys were counterbalanced across subjects for hand of response. In the identification and phonetic match tasks, RT feedback was presented to the subjects on an oscilloscope. During the physical match task, subjects were given both RT and corrective feedback. The feedback appeared immediately after a subject's response and remained on for $400 \mathrm{msec}$. If the subject did not respond within $905 \mathrm{msec}$ after timing began in the identification task or within $835 \mathrm{msec}$ after timing began in the phonetic and physical match tasks, an error message appeared on the scope.

Subjects were tested individually in two sessions lasting approximately $1 \frac{1 / 4}{h}$ each. Sessions were on separate days, and one experimental tape was presented at each session.

Subjects. The 16 subjects were paid recruits from the University of Oregon subject pool. All reported to be right-handed and were native English speakers. None of the subjects had had experience with synthesized speech prior to this experiment.

\section{Experiment 2}

Experiment materials. Stimuli were the 13 vor syllables used in Experiment 1. Two experimental tapes were prepared as described for the tapes in the first experiment, with the exception that ISIs were $250 \mathrm{msec}$ for matching trials instead of $570 \mathrm{msec}$. The ISI was shortened in this experiment in order to reduce possible memory loss for auditory information.

Procedure. There was no feedback given during test blocks in this experiment. For practice trials, however, feedback was given as described in Experiment 1. The subjects were given $1 \mathrm{sec}$ to respond in the identification task as well as in the matching tasks and were given an error message during practice blocks if they had not responded within the allotted time. Except for the manipulation in feedback presentation, instructions were the same as in Experiment 1.

Each subject listened to both experimental tapes on consecutive days. Order of tape presentation was counterbalanced across the subjects. Half the subjects were presented the identification and phonetic match tape followed by the physical match tape. The other half of the subjects received the physical match tape first. Response key was counterbalanced across subjects for hand as in Experiment 1.

Subjects. The subjects were eight paid volunteers recruited from the University of Oregon subject pool. All were right-handed native English speakers and had had no prior experience with synthesized speech.

\section{RESULTS}

\section{Identification Task}

The average identification function for the subjects in Experiment 1 is graphed in Figure 1. The identification function for the subjects in Experiment 2 is strikingly similar. ${ }^{1}$ In both experiments, the average boundary was between 25 - and 30 -msec VOT, which is in agreement with earlier studies (Lisker \& Abramson, 1970; Pisoni \& Tash, 1974). Included on the graph are mean RTs to identification. An analysis of variance on RTs to identification for Stimulus Value by Order (identification and phonetic match tasks first or physical match task first) in the two experiments revealed no significant main effects or interactions. The fact that RTs were not significantly longer for stimulus values at the phoneme boundary than at phoneme centers,

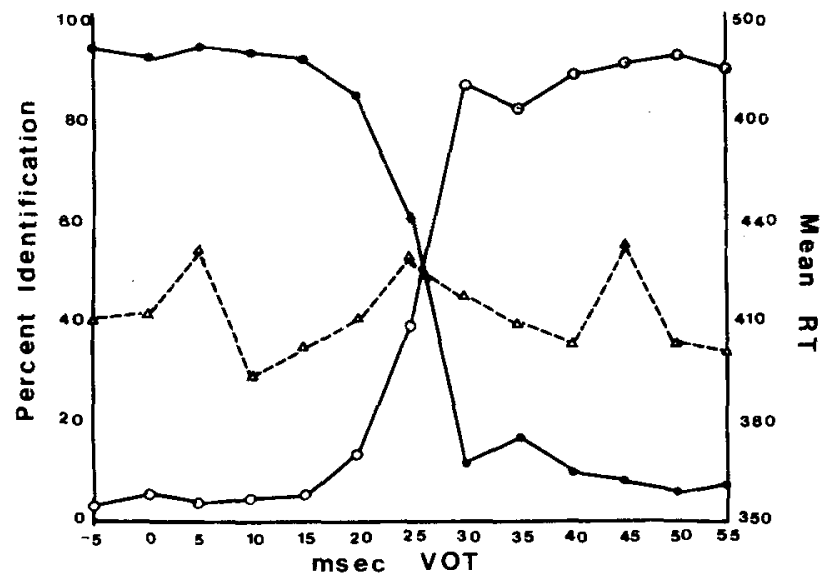

Figure 1. Identification function for Experiment 1. Also shown are the mean RTs to identification for each stimulus value.

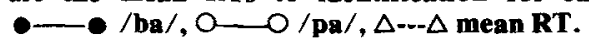

$F(12,168)=1.62$ and $F(12,72)=1.62$, for Experiments 1 and 2, respectively, suggests that subjects in the present study were not as influenced by acoustic information as have been listeners in previous studies (Pisoni \& Tash, 1974; Studdert-Kennedy, Liberman, \& Stevens, 1963).

Individual phoneme boundaries were determined for each subject. The lower boundary was defined as that VOT value at which all values less than or equal to that value were identified on at least $70 \%$ of the trials as $/ \mathrm{ba} /$. The upper phoneme boundary was that VOT value at which all values greater than or equal to the upper boundary were identified as /pa/ on at least $70 \%$ of the trials.

For two subjects in Experiment 2, these criteria for phoneme boundaries could not be met. Boundary stimuli for the two subjects were identified consistently at least $80 \%$ of the time. But for subject K.S., the $+5-\mathrm{msec}$ VOT stimulus was identified as $/ \mathrm{ba} /$ on only $50 \%$ of the test trials. Inspection of his practice block revealed that he had identified the stimulus as /ba/ on $80 \%$ of those practice trials. This stimulus was therefore included as a member of the $/ b /$ phoneme category for this subject. For subject J.F., the +40 -msec and $+45-\mathrm{msec}$ VOT stimuli were identified as /pa/ on only $60 \%$ of the trials. The decision was made to include these stimuli was representing the $/ p /$ phoneme for this subject.

For both the physical and phonetic match tasks, reference was made to these individual phoneme boundaries to determine if the stimulus pair on each trial belonged to the same or different phonetic categories for a given subject.

\section{Physical Match Task}

Individual abilities to make within-category discriminations in the physical match task were evaluated by determining a $\mathrm{d}^{\prime}$ score for each subject. 
Table 1

Individual Within-Categories Discrimination Scores

\begin{tabular}{|c|c|c|c|}
\hline \multicolumn{2}{|c|}{$\begin{array}{l}\text { Identification and Phonetic } \\
\text { Match Task First } \\
\end{array}$} & \multicolumn{2}{|c|}{$\begin{array}{c}\text { Physical Match } \\
\text { Task First }\end{array}$} \\
\hline Subject & d' Score & Subject & d' Score \\
\hline \multicolumn{4}{|c|}{ Experiment 1} \\
\hline $\begin{array}{l}\text { B.H. } \\
\text { N.L. } \\
\text { A.A. } \\
\text { D.B. } \\
\text { E.B. } \\
\text { Mk.B. } \\
\text { B.B. } \\
\text { D.W. }\end{array}$ & $\begin{array}{r}.34 \\
-.12 \\
.44 \\
.09 \\
.30 \\
.08 \\
.31 \\
.16\end{array}$ & $\begin{array}{l}\text { W.B. } \\
\text { T.K. } \\
\text { Mg.B. } \\
\text { P.B. } \\
\text { L.M. } \\
\text { R.F. } \\
\text { M.M. } \\
\text { My.B. }\end{array}$ & $\begin{array}{l}.81 \\
.28 \\
.30 \\
.81 \\
.79 \\
.38 \\
.60 \\
.90\end{array}$ \\
\hline Mean & .200 & & .609 \\
\hline \multicolumn{4}{|c|}{ Experiment 2} \\
\hline $\begin{array}{l}\text { M.S. } \\
\text { R.I. } \\
\text { D.C. } \\
\text { E.M. }\end{array}$ & $\begin{array}{l}.36 \\
.62 \\
.77 \\
.38\end{array}$ & $\begin{array}{l}\text { D.B. } \\
\text { J.B. } \\
\text { K.S. } \\
\text { J.F. }\end{array}$ & $\begin{array}{r}.94 \\
.84 \\
.96 \\
1.00\end{array}$ \\
\hline Mean & .532 & & .935 \\
\hline
\end{tabular}

"Hits" were defined as "same" responses for identity trials and "false alarms" were defined as "same" responses for same-category nonidentity trials. For purposes of this test, all nonidentity trials were averaged together. Individual $\mathrm{d}^{\prime}$ scores for the two orders of presentation are given in Table 1 for both experiments. A $t$ test comparing the two groups in Experiment 1 using the $\mathrm{d}^{\prime}$ scores indicated a large difference in the groups, $t(14)=3.04, p<.005$, with the group receiving the physical match task first making within-category discriminations more accurately than the group which received the identification and phonetic match tasks first. Likewise, the two groups in Experiment 2 were found to be statistically different, $t(6)=3.853, p<.01$.

A comparison of $\mathrm{d}^{\prime}$ scores in the two experiments revealed that within-category discrimination ability was superior in Experiment 2, $t(22)=2.654$, $p<.01$. Two explanations are possible. The first is that shorter ISIs made the discriminations easier because more auditory information from the first member of a stimulus pair was still available to subjects when the second stimulus was presented than was available with the longer ISIs. The second possible explanation is that within-category discriminations were superior in Experiment 2 because, with the eliminations of feedback, the subjects in the second experiment did not feel the pressure to respond quickly which the subjects in the first experiment felt. Reaction times in Experiment 2 were considerably longer than in Experiment 1. Accuracy, however, did not improve in Experiment 2 for the phonetic match task (21\% errors for Experiment 1 and $22 \%$ errors for Experiment 2). This lack of difference in accuracy scores suggests that the interpretation that subjects were sacrificing accuracy for speed is untenable.

Notice that the subjects in Experiment 2 and 5 subjects in Experiment 1 (My.B., W.B., P.B., L.M., and M.M.) had relatively high $\mathrm{d}^{\prime}$ scores. These results indicate that some listeners can directly use auditory cues which lead to phoneme identification. The data for the subjects in Experiment 2 and for the 5 subjects with the highest d' scores and the 11 subjects with low $d^{\prime}$ scores in Experiment 1 were analyzed separately. Percentage errors and mean RTs for correct responses were analyzed in the two matching tasks.

Results of the physical match task are given in Table 2.

Subjects in Experiment 1 with the highest d' scores. For these subjects, there was an average error rate of $31 \%$ on the physical match task. One-way ANOVAs on errors revealed an effect of steps $(0,1,3$, and 5 steps' acoustic difference) for same phonetic category trials, $F(3,12)=13.56, p<.001$, but no effect of steps $(1,3,5$ steps) for different-category trials, $F(2,8)=2.03$. There were many less errors on identity trials than on other same-category trials, $\mathrm{p}<.01$, as revealed by a Newman-Keuls test. Samecategory trials accounted for most of the errors. As indicated in a two-way analysis of variance, this category effect was significant, $F(1,4)=108.49$, $\mathrm{p}<.001$. The overall difference in one-, three-, and five-step trials was only marginally significant, $F(2,8)$ $=4.72, \mathrm{p}<.10$, with decreasing errors for larger physical discrepancies. There was no statistical

Table 2

Mean RT (in milliseconds) for Correct Responses in the Physical Match Task

\begin{tabular}{|c|c|c|c|c|c|c|}
\hline \multirow[b]{2}{*}{ Number of Steps } & \multicolumn{4}{|c|}{ Experiment 1} & \multicolumn{2}{|c|}{ Experiment 2} \\
\hline & Same & Different & Same & Different & Same & Different \\
\hline $\begin{array}{c}\text { Identity } \\
1 \\
3 \\
5\end{array}$ & $\begin{array}{l}515(17) \\
518(64) \\
522(48) \\
511(46)\end{array}$ & $\begin{array}{l}484(22) \\
498(12) \\
466(8)\end{array}$ & $\begin{array}{l}470(28) \\
485(66) \\
485(61) \\
494(52)\end{array}$ & $\begin{array}{l}483(27) \\
504(23) \\
485(19)\end{array}$ & $\begin{array}{l}655(17) \\
694(70) \\
697(57) \\
697(50)\end{array}$ & $\begin{array}{l}685(44) \\
678(15) \\
677(12)\end{array}$ \\
\hline
\end{tabular}

Note-Percent error is given in parentheses. 
difference in error rates on identity trials for these subjects under the two matching instructions. There were no significant RT effects for steps in the physical match task for either same or different phonetic category trials.

For RTs, the critical test for effect of the phoneme boundary approached significance in an overall two-way analysis of variance, $F(1,4)=7.59, p<.10$. Different phonetic category trials tended to be faster than same phonetic category trials. A serial process model would predict that there should be no effect of the phoneme boundary. While these results of these subjects are only suggestive that the boundary is influencing responding, there is another bit of evidence from these subjects which indicates that processing of auditory and phonetic information is not serial. A two-tailed correlated $t$ test comparing identity items in the phonetic and physical match tasks found that identity matches with phonetic instructions were faster than identity matches with physical match instructions, $\mathrm{t}(4)=3.572, \mathrm{p}<.05$. With phonetic match instructions, subjects could make decisions on the basis of either auditory or phonetic information. With physical match instructions, however, decisions could only be made on the basis of auditory information. The fact that redundant information speeded response time suggests that the information is being processed in parallel.

Subjects in Experiment 1 with low d' scores. Overall there were $42 \%$ errors for these subjects on the physical match task. On same-category trials, number of steps' acoustic difference was significant, $F(3,29)$ $=17.28, \mathrm{p}<.001$, with identity trials having less errors than other trials (Newman-Keuls, $\mathrm{p}<.01$ ). In an overall analysis of errors, steps and category were significant, $F(2,19)=25.34, p<.001$, and $F(1,9)$ $=41.75, \mathrm{p}<.001$, respectively. One-step trials had more errors than either three- or five-step trials (Newman-Keuls, $p<.01$ ), while three-step trials had more errors than five-step trials $(p<.05)$. There were more errors for same-category trials than different-category trials. There were no significant main effects or interactions found in analyses of the RT data. The phoneme boundary did not influence response time for these subjects, $F(1,9)=1.85$. For these subjects, crossing the phonetic boundary did not speed RT.

There were more errors on identity trials with physical match instructions than with phonetic match instructions, $t(10)=4.079, p<.005$. Reaction times to identity trials under the two instructions, however, were not statistically different, $\mathrm{t}(10)=.646$.

Experiment 2. These subjects were influenced by the phonetic boundary, $F(1,6)=22.09, p<.005$, with same-category trials slower than differentcategory trials. As with the five subjects in Experiment 1 who had high d' scores, phonetic informa- tion was influencing these subjects when making auditory decisions. This is a result that is not consistent with a serial process model of auditory and phonetic processing. In addition, a correlated test performed on RTs for identity items for the phonetic and physical match tasks revealed that matches under the physical match instructions were slower than matches under phonetic instructions, $t(7)=2.536$, $\mathrm{p}<.05$. Again, this result is inconsistent with a serial model. Errors for identity items in the two tasks were not significantly different, $t(7)=1.659$.

One-way ANOVAs on same- and different-category RTs showed no significant differences. Error data exhibited reliable differences of steps, however, for both same, $F(3,18)=13.50, p<.001$, and different category items, $F(2,10)=25.31, p<.001$.Identity trials had fewer errors than one-, three-, and five-step same-category trials, $p<.01$. One-step different-category trials had more errors than threeor five-step trials, $p<.05$. Analysis of overall errors showed significant main effects of steps, $F(2,12)$ $=30.93, \mathrm{p}<.001$, and category, $\mathrm{F}(1,6)=75.58$, $p<.001$. One-step trials had more errors than threeand five-step trials, $p<.01$, as revealed in a NewmanKeuls analysis. There were more errors for samecategory than for different-category trials. Steps interacted with category, $F(2,10)=4.88, p<.05$, with a differential rate in decline of same and different phonetic category errors as physical similarity decreased. The overall error rate was $37 \%$.

\section{Phonetic Match Task}

Results of the phonetic match task are given in Table 3.

Subjects in Experiment 1 with the highest d' scores. One-way ANOVAs on errors revealed significant main effects of steps for both same- and differentcategory trials, $\mathrm{F}(3,12)=4.16, \mathrm{p}<.05$, and $\mathrm{F}(3,12)$ $=9.43, \mathrm{p}<.005$, respectively. For same-category trials, there were fewer errors for identity trials than for other trials, $p<.05$, and for different-category trials more errors occurred on one-step trials than on three-, five- and seven-step trials, $p<.01$, as shown by Newman-Keuls analyses. Overall, there was a main effect of steps, $F(2,8)=8.52, p<.025$, with a Newman-Keuls analysis showing more errors for one-step trials than for three- and five-step trials, $\mathrm{p}<.05$. Steps interacted with category, $F(2,8)=9.96$, $\mathrm{p}<.01$. There was an average of $21.5 \%$ errors.

ANOVAs on RTs indicated a main effect of steps on same-category trials, $F(3,12)=4.18, p<.05$, as well as a marginal effect of steps on differentcategory trials, $F(3,12)=2.63, p<.10$. A NewmanKeuls test indicated that identity items were faster than five-step trials, $\mathrm{p}<.05$.

For these subjects, the effects expected on the basis of the results of Pisoni and Tash (1974) were 
Table 3

Mean RT (in milliseconds) for Correct Responses in the Phonetic Match Task

\begin{tabular}{|c|c|c|c|c|c|c|}
\hline \multirow[b]{4}{*}{ Number of Steps } & \multicolumn{4}{|c|}{ Experiment 1} & \multirow{2}{*}{\multicolumn{2}{|c|}{ Experiment 2}} \\
\hline & \multicolumn{2}{|c|}{$\begin{array}{l}\text { Subjects with the } \\
\text { Highest d' Scores }\end{array}$} & \multicolumn{2}{|c|}{$\begin{array}{c}\text { Subjects with Low } \\
\text { d' Scores }^{\prime}\end{array}$} & & \\
\hline & \multicolumn{2}{|c|}{ Phonetic Category } & \multicolumn{2}{|c|}{ Phonetic Category } & \multicolumn{2}{|c|}{ Phonetic Category } \\
\hline & Same & Different & Same & Different & Same & Different \\
\hline $\begin{array}{c}\text { Identity } \\
1 \\
3 \\
5 \\
7\end{array}$ & $\begin{array}{l}452(14) \\
462(21) \\
467(21) \\
481(26)\end{array}$ & $\begin{array}{l}546(54) \\
462(17) \\
453(11) \\
446(7)\end{array}$ & $\begin{array}{l}464(16) \\
469(21) \\
461(18) \\
471(27)\end{array}$ & $\begin{array}{l}482(31) \\
505(26) \\
485(17) \\
478(12)\end{array}$ & $\begin{array}{l}549(8) \\
601(23) \\
606(22) \\
609(25)\end{array}$ & $\begin{array}{l}686(62) \\
630(23) \\
625(18) \\
610(10)\end{array}$ \\
\hline
\end{tabular}

Note-Percent error is given in parentheses.

obtained for both errors and RTs. When phonetically matching, as similarity in pairs decreased, samecategory RTs and errors increased and differentcategory RTs and errors decreased. These subjects, who were able to deliberately use auditory cues as shown by their $d^{\prime}$ scores, were also indirectly influenced by these cues as revealed in this phonetic match task.

Subjects in Experiment 1 with low $d^{\prime}$ scores. Analysis of the errors found main effects of steps for both same phonetic category, $\mathrm{F}(3,28)=2.99, \mathrm{p}<.05$ and different-category trials, $F(3,19)=6.43$, $p<$ .005 . For same-category trials, five-step trials had more errors than either identity $(p<.01)$ or one-step trials $(\mathrm{p}<.05)$, as revealed by a Newman-Keuls analysis. The post hoc analysis of different-category trials indicated that one-step trials had more errors than five- and seven-step trials, $p<.05$ and $p<.01$, respectively. The overall analysis of one-, three-, and five-step trials for both same and different phonetic category errors revealed no main effects or interaction of Steps by Category. The overall error rate was $20 \%$. Analyses of RTs in the phonetic match task found no significant effects. These subjects, who showed little ability to make direct use of auditory cues, were not influenced by acoustic similarity in this task either. The performance of these subjects on the phonetic match task contrasts sharply with the performance on this task of the subjects with the highest $d^{\prime}$ scores.

Experiment 2. Results of Experiment 2 are very similar to those for the five subjects with the highest d' scores in Experiment 1. The subjects in the second experiment generally had high $d^{\prime}$ scores on the physical match task, and results indicated that auditory information influenced them when matching phonetically.

Errors revealed that there were main effects of steps for both same-category trials, $F(3,18)=8.24$, $p<.005$, and different-category trials, $F(3,16)$ $=31.82, p<.001$. Indications from a NewmanKeuls analysis were that identity trials had fewer errors than one-, three-, and five-step trials, $\mathrm{p}<.01$.
For different-category trials, there were more errors on one-step than on three-, five-, and seven-step trials, $\mathrm{p}<.01$, and more errors on three-step than on seven-step trials, $p<.05$. An overall three-way analysis of the phonetic match task for errors found a main effect of steps, $F(2,12)=9.10, p<.005$, as well as an interaction of steps and category, $F(2,10)=12.28, p<.005$. The main effect of steps reflected more errors for one-step trials than for three- and five-step trials, as shown by Newman-Keuls comparisons. Different-category matches mainly contributed to the error rate for these stimuli. Average errors for this phonetic match task were $22 \%$.

Analyses of this task also indicated that steps were not significant for RTs to same-category items, $F(3,18)=.59$, but were significant for differentcategory trials, $F(3,16)=3.99, p<.05$. One-step trials were slower than three- and five-step trials $(p<.05)$ and seven-step trials $(p<.01)$, as shown by a Newman-Keuls test. For RTs, an overall analysis showed only a main effect of category, $F(1,6)=$ $14.32, \mathrm{p}<.01$, reflecting the fact that it took longer to respond "different" than "same."

\section{DISCUSSION}

In this study, subjects identified the synthetic speech, as have listeners in previous studies (Lisker \& Abramson, 1970; Pisoni \& Tash, 1974). Thus, the stimuli were phonetically heard by these subjects as by other listeners. But the present subjects did not show the degree of RT increase at phoneme boundaries which had been observed in earlier studies (Pisoni \& Tash, 1974; Studdert-Kennedy et al., 1963). The finding that the present subjects were not as influenced by acoustic cues may be due to the fact that subjects in the current study were not selected. The listeners used by Pisoni and Tash (1974), for example, all had had previous experience with synthesized speech. Prior experience with these stimuli may be a critical factor. 


\section{Use of Acoustic Cues in Speech Perception}

The present study found that low-level acoustic information can be deliberately used by subjects in discriminating speech. Also, in agreement with Pisoni and Tash (1974), auditory information was found to affect phonetic judgments. However, not all subjects in all conditions used the acoustic cues equally well.

The present study identified two factors which were relevant in determining the degree to which within-category auditory information can be used. ${ }^{2}$ One factor, in agreement with Pisoni and Lazarus (1974), was prior experience with synthetic speech. Listeners whose first experience with synthetic speech was in the identification task had more difficulty making within-category discriminations than listeners who did not have this labeling experience prior to the physical match task.

The second factor determining ability to make within-category discriminations was the duration of the ISI. Subjects were generally less able to use auditory information at long ISIs $(570 \mathrm{msec})$ than at short ISIs $(250 \mathrm{msec})$. This result is in contrast to Pisoni (1973), who found no difference for withincategory discrimination ability at ISIs varying from 0 to $2.0 \mathrm{sec}$. It is possible that this difference can be explained by examining the instructions given to subjects in the two studies. Subjects in the physical match task in the present study were informed that the two syllables of a trial could represent the same CV syllable yet not be physically identical. Thus, they were looking for auditory distinctions. The subjects in Pisoni's (1973) experiment were only told to decide whether the stimuli were the "same" or "different" and were not informed that two syllables from the same category could be acoustically different. Pisoni and Lazarus (1974) have found that instructions can affect listeners' ability to use auditory information.

\section{Individual Differences}

The subjects in Experiment 2 and some subjects in Experiment 1 made within-category discriminations fairly well in the physical match task, while other subjects in Experiment 1 were little able to use auditory cues. Some of these differences appear to be due to effects of order of task presentation (physical match task first or identification and phonetic match tasks first), but not all differences can be attributable to this factor. Within each order of presentation, there is a wide range of individual differences. Subjects who had relatively high $\mathrm{d}^{\prime}$ scores were able to deliberately distinguish speech sounds solely on the basis of auditory cues. These subjects also showed greater influence of auditory information in their responding in the phonetic match task than subjects who were poor at making within-category discriminations. Apparently, subjects who were able to deliber- ately use auditory cues also used these cues implicitly when making phonetic judgments. Conversely, subjects who could not deliberately use these cues also did not implicitly use them when making phonetic judgments.

Possibly the reason some subjects were more influenced by auditory information than other subjects had to do with the degree to which subjects can retain information about stop consonants in acoustic memory. Crowder and Morton (1969) presented evidence for a precategorical acoustic store (PAS) which could retain acoustic information for as long as $2 \mathrm{sec}$. In further experiments, however, Crowder $(1971,1973)$ found that stop consonants were not well retained in PAS.

Application of the ideas of Crowder $(1971,1973)$ could perhaps be used to account for the individual differences in the present study. Consonant information may remain in PAS longer for some subjects than for others. For the subjects who could make within-category discrimination, some acoustic information was still available to the subject at the time of presentation of the second syllable of a trial. Consonant information may have been more resistant to degradation for these subjects than it was for subjects with low d' scores. For the subjects who could make within-category discriminations, both auditory and phonetic information would have been available on each trial. These subjects would have been expected to show effects of the auditory information in the phonetic match task as well as in ability to make within-category discriminations. It would also be expected that the phoneme boundary would affect these subjects in the physical match task because redundant information would be presented on different phonetic category trials but not on same phonetic category trials. And, in fact, subjects who were able to use auditory information (as defined by high within-category d' scores) did show the expected effects in phonetic matches and were affected by the phoneme boundary in the physical match task. Conversely, subjects for whom consonant information was rapidly degraded would not be able to make within-category discriminations and would not show an effect of crossing the phoneme boundary, because they would not have redundant information available to them. Only phonetic information would be available to these subjects when making decisions. Indeed, subjects with low d' scores did not demonstrate any redundancy gain.

\section{Processing of Auditory and Phonetic Information}

The present study indicates that decisions about auditory and phonetic information are being carried out in parallel. For subjects who showed ability to use auditory information, phonetic information influenced decisions in the physical match task. The time necessary to respond "different" for same 
phonetic category nonidentity trials in the physical match task was longer than the time necessary to respond "different" for different phonetic category trials. Also, response times were faster for identity trials with phonetic match instructions than with physical match instructions. The redundant information in these two cases speeded response time. A serial process model, such as that of Pisoni and Tash (1974), predicts that there should be no RT differences in the two conditions because auditory decisions would always be made before phonetic decisions. According to that model, therefore, phonetic information should not affect the time necessary to make auditory decisions; only with some type of parallel process model would there be redundancy gain.

\section{REFERENCES}

BARCLAY, J. R. Noncategorical perception of a voiced stop: A replication. Perception \& Psychophysics, 1972, 11, 269-273.

Crowder, R. G. The sounds of vowels and consonants in immediate memory. Journal of Verbal Learning and Verbal Behavior, 1971, 10, 587-597.

CrowDer, R. G. Representation of speech sounds in precategorical acoustic storage. Journal of Experimental Psychology, 1973, 1, 14-24.

Crowder, R. G., \& Morton, J. Precategorical acoustic storage (PAS). Perception \& Psychophysics, 1969, 5, 365-373.

Hanson, V. L. Within category discriminations in speech perception. Unpublished master's thesis, University of Oregon, Eugene, 1976.

Liberman, A. M., Cooper, F. S., Shankweiler, D. S., \& Studdert-Kennedy, M. Perception of the speech code. Psychological Review, 1967, 74, 431-461.

Lib erman, A. M., Harris, K. S., Hoffman, H. S., \& Griffith, B. C. The discrimination of speech sounds within and across phoneme boundaries. Journal of Experimental Psychology, 1957, 54, 358-368:
Lusker, L., \& Abramson, A. S. The voicing dimension: Some experiments in comparative phonetics. In Proceedings of the Sixth International Congress of Phonetic Sciences, Prague, 1967. Prague: Academia, 1970. Pp. 563-567.

Pison, D. B. On the nature of categorical perception of speech sounds. Doctoral thesis, University of Michigan, 1971.

Pisoni, D. B. Auditory and phonetic memory codes in the discrimination of consonants and vowels. Perception \& Psychophysics, 1973, 13, 253-260.

Pisoni, D. B., \& Lazarus, J. H. Categorical and noncategorical modes of speech perception along the voicing continuum. Journal of the Acoustical Society of America, 1974, 55, 328-333.

Prson, D. B., \& TAsh, J. Reaction times to comparisons within and across phonetic categories. Perception \& Psychophysics, 1974, 15, 285-290.

Studdert-Kennedy, M., Liberman, A. M., \& Stevens, K. N. Reaction time to synthetic stop consonants and vowels at phoneme centers and at phoneme boundaries. Journal of the Acoustical Society of America, 1963, 35, 1900.

Wood, C. C. Parallel processing of auditory and phonetic information in speech perception. Perception \& Psychophysics, $1974,15,501-508$.

\section{NOTES}

1. Two of the original eight subjects in Experiment 2 yielded inconsistent identification functions. There was no indication that they could discriminate the synthesized syllables. Both of these subjects were in the experimental group which received the physical match task first. Two additional subjects were tested to replace these two subjects.

2. A third factor affecting within-category discrimination ability was reported in Hanson (1976). It was found that subjects were better able to make within-category discriminations for the bilabial stops described in the present study than for voiced stops differing in place of articulation.

(Received for publication August 2, 1976; revision accepted January 15,1977 .) 